\title{
Representations of integers as sums of primes from a Beatty sequence
}

by

\author{
William D. Banks, Ahmet M. GüloĞLU \\ and C. Wesley Nevans (Columbia, MO)
}

1. Introduction. The celebrated 1937 theorem of Vinogradov states that every sufficiently large odd number is the sum of three prime numbers. However, the statement is no longer true if all three primes are required to lie in the Beatty sequence

$$
\mathcal{B}_{\alpha}=\{\lfloor\alpha m\rfloor: m \in \mathbb{N}\}
$$

for a fixed irrational number $\alpha>3$. Indeed, if $N$ is odd and

$$
N=\left\lfloor\alpha m_{1}\right\rfloor+\left\lfloor\alpha m_{2}\right\rfloor+\left\lfloor\alpha m_{3}\right\rfloor
$$

for some $m_{1}, m_{2}, m_{3} \in \mathbb{N}$, it is easy to see that

$$
N \alpha^{-1} \leq m_{1}+m_{2}+m_{3}<N \alpha^{-1}+3 \alpha^{-1} .
$$

Hence, the relation (1) cannot hold if the fractional part $\left\{N \alpha^{-1}\right\}$ of $N \alpha^{-1}$ lies in the open interval $\left(0,1-3 \alpha^{-1}\right)$, which happens for about $\frac{1}{2}\left(1-3 \alpha^{-1}\right) X$ positive odd integers $N \leq X$. On the other hand, for an irrational number $\alpha$ of finite type (see Section 2.2) in the range $1<\alpha<3$, we show that every sufficiently large odd number is the sum of three prime numbers, each of which lies in the Beatty sequence $\mathcal{B}_{\alpha}$.

More generally, for fixed $\alpha, \beta \in \mathbb{R}$ with $\alpha>1$, we study the problem of representing integers as sums of primes from the non-homogeneous Beatty sequence

$$
\mathcal{B}_{\alpha, \beta}=\{n \in \mathbb{N}: n=\lfloor\alpha m+\beta\rfloor \text { for some } m \in \mathbb{Z}\} .
$$

In this paper, we prove the following:

TheOREM 1. Let $\alpha, \beta \in \mathbb{R}$ with $\alpha>1$, and suppose that $\alpha$ is irrational and of finite type. Then:

2000 Mathematics Subject Classification: 11P32, 11B50.

Key words and phrases: Beatty sequence. 
(i) Almost all even numbers $N$ can be expressed as the sum of two primes from the Beatty sequence $\mathcal{B}_{\alpha, \beta}$ if and only if $\alpha<2$.

(ii) For any integer $\kappa \geq 3$, every sufficiently large number $N \equiv \kappa(\bmod 2)$ can be expressed as the sum of $\kappa$ primes from the Beatty sequence $\mathcal{B}_{\alpha, \beta}$ if and only if $\alpha<\kappa$.

To state our results more explicitly, we define for every integer $\kappa \geq 2$ the function

$$
\mathcal{G}_{\kappa}(N)=\mathcal{G}_{\kappa}(\alpha, \beta ; N)=\sum_{\substack{n_{1}+\cdots+n_{\kappa}=N \\ n_{1}, \ldots, n_{\kappa} \in \mathcal{B}_{\alpha, \beta}}} \Lambda\left(n_{1}\right) \cdots \Lambda\left(n_{\kappa}\right) \quad(N \geq 1),
$$

where $\Lambda$ is the von Mangoldt function:

$$
\Lambda(n)= \begin{cases}\log p & \text { if } n \text { is a positive power of the prime } p \\ 0 & \text { otherwise }\end{cases}
$$

By partial summation, our estimates for $\mathcal{G}_{\kappa}(N)$ lead to estimates for the number of representations of an integer $N \equiv \kappa(\bmod 2)$ as the sum of $\kappa$ primes from the Beatty sequence $\mathcal{B}_{\alpha, \beta}$.

Let $\psi=\psi_{\alpha}$ be the periodic function with period one which is defined on the interval $(0,1]$ as follows:

$$
\psi(x)= \begin{cases}1 & \text { if } 0<x \leq \alpha^{-1} \\ 0 & \text { if } \alpha^{-1}<x \leq 1\end{cases}
$$

The function $\psi$ is closely related to the characteristic function of the set $\mathcal{B}_{\alpha, \beta}$. Let $\psi^{(1)}=\psi$, and for every $\kappa \geq 2$, let $\psi^{(\kappa)}$ denote the $\kappa$-fold convolution of $\psi$ with itself, defined inductively by

$$
\psi^{(\kappa)}(x)=\int_{0}^{1} \psi^{(\kappa-1)}(x-y) \psi(y) d y \quad(\kappa \geq 2) .
$$

Finally, for every $\kappa \geq 2$ we define the singular series

$$
\mathfrak{S}_{\kappa}(N)=\prod_{p \mid N}\left(1+\frac{(-1)^{\kappa}}{(p-1)^{\kappa-1}}\right) \prod_{p \nmid N}\left(1+\frac{(-1)^{\kappa+1}}{(p-1)^{\kappa}}\right) \quad(N \geq 1) .
$$

The numbers $\mathfrak{S}_{\kappa}(N)$ arise naturally in estimates for the number of representations of an integer as a sum of $\kappa$ prime numbers. Note that $\mathfrak{S}_{\kappa}(N)=0$ if and only if $N \not \equiv \kappa(\bmod 2)$.

Theorem 2. Let $\alpha, \beta \in \mathbb{R}$ with $\alpha>1$, and suppose that $\alpha$ is irrational and of finite type. Then, for any constant $C>0$, the estimate

$$
\mathcal{G}_{2}(N)=\psi^{(2)}(\gamma N+2 \delta) \mathfrak{S}_{2}(N) N+O\left(\frac{N}{(\log N)^{C}}\right)
$$

holds for all but $O\left(X(\log X)^{-C}\right)$ integers $N \leq X$, where $\gamma=\alpha^{-1}, \delta=$ $\alpha^{-1}(1-\beta)$, and the implied constants depend only on $\alpha$ and $C$. 
ThEOREM 3. Let $\alpha, \beta \in \mathbb{R}$ with $\alpha>1$, and suppose that $\alpha$ is irrational and of finite type. Then, for every integer $\kappa \geq 3$ and any constant $C>0$,

$$
\mathcal{G}_{\kappa}(N)=\psi^{(\kappa)}(\gamma N+\kappa \delta) \mathfrak{S}_{\kappa}(N) \frac{N^{\kappa-1}}{(\kappa-1) !}+O\left(\frac{N^{\kappa-1}}{(\log N)^{C}}\right)
$$

where $\gamma=\alpha^{-1}, \delta=\alpha^{-1}(1-\beta)$, and the implied constant depends only on $\alpha, \kappa$ and $C$.

The proof of Theorem 2 is given in Section 3 (see the Remark after the statement of Theorem 4) and that of Theorem 3 is given in Section 4 (see the Remark after the statement of Proposition 1). In Section 5 we study properties of the convolutions $\psi^{(\kappa)}(\kappa \geq 2)$ and, in particular, derive a sharp lower bound for values of $\psi^{(\kappa)}$ in the special case that $\kappa=\lceil\alpha\rceil$. Our proof of Theorem 1, which is given in Section 6, follows immediately from the results of Section 5.

Our arguments have been strongly influenced by the treatment of the Goldbach problem that is given in the book [6] of Iwaniec and Kowalski, and we adopt a similar notation here. Our underlying approach relies heavily on ideas from a recent paper of Banks and Shparlinski [2] on primes in a Beatty sequence.

Acknowledgements. The authors wish to thank Ignacio Uriarte-Tuero and Bob Vaughan for helpful conversations. This work was done entirely at the University of Missouri-Columbia; the support of this institution is gratefully acknowledged.

\section{Preliminaries}

2.1. Notation. The symbol $\llbracket x \rrbracket$ is used to denote the distance from the real number $x$ to the nearest integer; that is,

$$
\llbracket x \rrbracket=\min _{n \in \mathbb{Z}}|x-n| \quad(x \in \mathbb{R}) .
$$

We denote by $\lfloor x\rfloor,\lceil x\rceil$ and $\{x\}$ the greatest integer $\leq x$, the least integer $\geq x$, and the fractional part of $x$, respectively. We also put $\mathbf{e}(x)=e^{2 \pi i x}$ for all $x \in \mathbb{R}$. As usual, we use $\mu$ and $\varphi$ to denote the Möbius and Euler functions, respectively.

Throughout the paper, the implied constants in symbols $O, \ll$ and $\gg$ may depend (where obvious) on the parameters $\alpha, \kappa, C$ but are absolute otherwise. We recall that for functions $F$ and $G$ the notations $F \ll G$, $G \gg F$ and $F=O(G)$ are all equivalent to the statement that the inequality $|F| \leq c|G|$ holds for some constant $c>0$.

2.2. Discrepancy of fractional parts. Recall that the discrepancy $D(M)$ of a sequence of (not necessarily distinct) real numbers $a_{1}, \ldots, a_{M} \in[0,1$ ) 
is defined by

$$
D(M)=\sup _{\mathcal{I} \subseteq[0,1)}\left|\frac{V(\mathcal{I}, M)}{M}-\right| \mathcal{I}||
$$

where the supremum is taken over all subintervals $\mathcal{I}=(c, d)$ of the interval $[0,1), V(\mathcal{I}, M)$ is the number of positive integers $m \leq M$ such that $a_{m} \in \mathcal{I}$, and $|\mathcal{I}|=d-c$ is the length of $\mathcal{I}$.

For any irrational number $\gamma$ we define its type $\tau$ by the relation

$$
\tau=\sup \left\{t \in \mathbb{R}: \liminf _{n \rightarrow \infty} n^{t} \llbracket \gamma n \rrbracket=0\right\} .
$$

Using Dirichlet's approximation theorem, it is easily seen that $\tau \geq 1$ for every irrational number $\gamma$. The well known theorems of Khinchin [7] and of Roth $[13,14]$ assert that $\tau=1$ for almost all real numbers (in the sense of the Lebesgue measure) and all irrational algebraic numbers $\gamma$, respectively; see also $[3,15]$.

For every irrational number $\gamma$, it is known that the sequence of fractional parts $\{\gamma\},\{2 \gamma\},\{3 \gamma\}, \ldots$, is uniformly distributed modulo 1 (for instance, see [9, Chapter 1, Example 2.1]). When $\gamma$ is of finite type, this statement can be made more precise. Let $D_{\gamma, \delta}(M)$ denote the discrepancy of the sequence of fractional parts $(\{\gamma m+\delta\})_{m=1}^{M}$. By [9, Chapter 2, Theorem 3.2] we have:

LEMMA 1. Let $\gamma$ be a fixed irrational number of finite type $\tau<\infty$. Then for all $\delta \in \mathbb{R}$ the following bound holds:

$$
D_{\gamma, \delta}(M) \leq M^{-1 / \tau+o(1)} \quad(M \rightarrow \infty),
$$

where the function implied by $o(\cdot)$ depends only on $\gamma$.

2.3. Numbers in a Beatty sequence. The following elementary result characterizes the set of numbers that occur in the Beatty sequence $\mathcal{B}_{\alpha, \beta}$ :

LEMma 2. Let $\alpha, \beta \in \mathbb{R}$ with $\alpha>1$, and put $\gamma=\alpha^{-1}, \delta=\alpha^{-1}(1-\beta)$. Then $n=\lfloor\alpha m+\beta\rfloor$ for some integer $m$ if and only if $0<\{\gamma n+\delta\} \leq \gamma$.

2.4. Estimates with the von Mangoldt function. The estimate below follows immediately from the Siegel-Walfisz theorem (see, for example, the book [5] by Huxley) using partial summation:

Lemma 3. Let $\kappa \geq 1$ be fixed. Then for any fixed constant $A>0$ and uniformly for integers $N \geq 3$ and $0 \leq c<d \leq(\log N)^{A}$ with $\operatorname{gcd}(c, d)=1$,

$$
\sum_{\substack{n \leq N \\ n \equiv c(\bmod d)}} \Lambda(n)(N-n)^{\kappa-1}=\frac{N^{\kappa}}{\kappa \varphi(d)}+O\left(N^{\kappa} \exp \left(-B(\log N)^{1 / 2}\right)\right),
$$

where $B>0$ is a constant that depends only on $\kappa$ and $A$.

We also need the following: 
Lemma 4. Let $\kappa \geq 1$ be fixed. For an arbitrary real number $\theta$ and coprime integers $c, d$ with $0 \leq c<d$, if $|\theta-a / b| \leq 1 / N$ and $\operatorname{gcd}(a, b)=1$, then

$\sum_{\substack{n \leq N \\ n \equiv c(\bmod d)}} \Lambda(n) \mathbf{e}(\theta n)(N-n)^{\kappa-1} \ll\left(b^{-1 / 2} N^{\kappa}+b^{1 / 2} N^{\kappa-1 / 2}+N^{\kappa-1 / 5}\right)(\log N)^{3}$, where the implied constant depends only on $\kappa$.

Proof. The special case $\kappa=1$ is a simplified and weakened version of a theorem of Balog and Perelli [1] (see also [11]), and the general case follows by partial summation.

2.5. The singular series. For every integer $\kappa \geq 2$, it is easy to check that the singular series

$$
\mathfrak{S}_{\kappa}(N)=\prod_{p \mid N}\left(1+\frac{(-1)^{\kappa}}{(p-1)^{\kappa-1}}\right) \prod_{p \nmid N}\left(1+\frac{(-1)^{\kappa+1}}{(p-1)^{\kappa}}\right)
$$

satisfies the identity

$$
\mathfrak{S}_{\kappa}(N)=\sum_{d \mid N} \sum_{\substack{c \geq 1 \\ \operatorname{gcd}(c, d)=1}} \frac{\mu(c)^{\kappa+1} \mu(d)^{\kappa} d}{\varphi(c)^{\kappa} \varphi(d)^{\kappa}}
$$

and for every $\kappa \geq 3$ we also have

$$
\mathfrak{S}_{\kappa}(N)=\sum_{\substack{c, d \geq 1 \\ \operatorname{gcd}(d, c N)=1}} \frac{\mu(c)^{\kappa} \mu(d)^{\kappa+1} d}{\varphi(c)^{\kappa-1} \varphi(d)^{\kappa}} .
$$

We moreover have the bound

$$
\mathfrak{S}_{2}(N) \ll \log \log N
$$

and for every $\kappa \geq 3$,

$$
\mathfrak{S}_{\kappa}(N) \ll 1
$$

3. Two Beatty primes. Fix $\alpha, \beta \in \mathbb{R}$ with $\alpha>1$, and suppose that $\alpha$ is irrational and of finite type. In this section, we focus our attention on the function

$$
\mathcal{G}_{2}(N)=\sum_{\substack{n_{1}+n_{2}=N \\ n_{1}, n_{2} \in \mathcal{B}_{\alpha, \beta}}} \Lambda\left(n_{1}\right) \Lambda\left(n_{2}\right) \quad(N \geq 1)
$$

Put $\gamma=\alpha^{-1}$ and $\delta=\alpha^{-1}(1-\beta)$, and let $\tau$ denote the (finite) type of $\gamma$. We recall that $\psi$ is the periodic function with period one which is defined by (2) on the interval $(0,1]$, and $\psi^{(2)}=\psi * \psi$ is the convolution of $\psi$ with itself. 
TheOREM 4. For any complex numbers $c_{N}$ and any constant $C>0$,

$$
\sum_{N \leq X} c_{N} \mathcal{G}_{2}(N)=\sum_{N \leq X} c_{N} \psi^{(2)}(\gamma N+2 \delta) \mathfrak{S}_{2}(N) N+O\left(\|c\|_{2} \frac{X^{3 / 2}}{(\log X)^{C}}\right)
$$

where $\|c\|_{2}=\left(\sum_{N \leq X}\left|c_{N}\right|^{2}\right)^{1 / 2}$.

REMARK. This result immediately yields a proof of Theorem 2. Indeed, taking $c_{N}=\mathcal{G}_{2}(N)-\psi^{(2)}(\gamma N+2 \delta) \mathfrak{S}_{2}(N) N$, we derive the bound

$$
\sum_{N \leq X}\left(\mathcal{G}_{2}(N)-\psi^{(2)}(\gamma N+2 \delta) \mathfrak{S}_{2}(N) N\right)^{2} \ll \frac{X^{3}}{(\log X)^{2 C}},
$$

and Theorem 2 follows at once.

Proof of Theorem 4. By Lemma 2 and the definition (2), it follows that

$$
\mathcal{G}_{2}(N)=\sum_{n_{1}+n_{2}=N} \Lambda\left(n_{1}\right) \Lambda\left(n_{2}\right) \psi\left(\gamma n_{1}+\delta\right) \psi\left(\gamma n_{2}+\delta\right) .
$$

According to a classical result of Vinogradov (see [16, Chapter I, Lemma 12]), for any $\Delta$ such that

$$
0<\Delta<\frac{1}{8} \quad \text { and } \quad \Delta \leq \frac{1}{2} \min \{\gamma, 1-\gamma\}
$$

there is a real-valued function $\Psi$ with the following properties:

(i) $\Psi$ is periodic with period one;

(ii) $0 \leq \Psi(x) \leq 1$ for all $x \in \mathbb{R}$;

(iii) $\Psi(x)=\psi(x)$ if $\Delta \leq\{x\} \leq \gamma-\Delta$ or if $\gamma+\Delta \leq\{x\} \leq 1-\Delta$;

(iv) $\Psi$ can be represented as a Fourier series

$$
\Psi(x)=\sum_{k \in \mathbb{Z}} g(k) \mathbf{e}(k x),
$$

where $g(0)=\gamma$, and the Fourier coefficients satisfy the uniform bound

$$
|g(k)| \ll \min \left\{|k|^{-1},|k|^{-2} \Delta^{-1}\right\} \quad(k \neq 0) .
$$

From the properties (i)-(iii) above, it follows that the estimate

$$
\Psi^{(2)}(x)=\psi^{(2)}(x)+O(\Delta)
$$

holds uniformly for all $x \in \mathbb{R}$, where $\Psi^{(2)}$ is the convolution $\Psi * \Psi$.

From (8) we see that

$$
\begin{aligned}
\mathcal{G}_{2}(N)= & \sum_{n_{1}+n_{2}=N} \Lambda\left(n_{1}\right) \Lambda\left(n_{2}\right) \Psi\left(\gamma n_{1}+\delta\right) \Psi\left(\gamma n_{2}+\delta\right) \\
& +O\left(V(\mathcal{I}, N)(\log N)^{2}\right)
\end{aligned}
$$


where $V(\mathcal{I}, N)$ is the number of positive integers $n \leq N$ such that

$$
\{\gamma n+\delta\} \in \mathcal{I}=[0, \Delta) \cup(\gamma-\Delta, \gamma+\Delta) \cup(1-\Delta, 1) .
$$

Since $|\mathcal{I}|=4 \Delta$, it follows from the definition (3) and Lemma 1 that

$$
V(\mathcal{I}, N) \ll \Delta N+N^{1-1 /(2 \tau)} .
$$

Now let $K \geq \Delta^{-1}$ be a large real number (to be specified later), and let $\Psi_{K}$ be the trigonometric polynomial given by

$$
\Psi_{K}(x)=\sum_{|k| \leq K} g(k) \mathbf{e}(k x) .
$$

Using (9), we see that the estimate

$$
\Psi_{K}(x)=\Psi(x)+O\left(K^{-1} \Delta^{-1}\right)
$$

holds uniformly for all $x \in \mathbb{R}$, and therefore

$$
\Psi_{K}^{(2)}(x)=\Psi^{(2)}(x)+O\left(K^{-1} \Delta^{-1}\right)=\psi^{(2)}(x)+O\left(\Delta+K^{-1} \Delta^{-1}\right),
$$

where we have used (10) in the second step. From the definition (13) we also have

$$
\Psi_{K}^{(2)}(x)=\sum_{|k| \leq K} g(k)^{2} \mathbf{e}(k x) .
$$

Inserting the estimate (14) into (11) and taking into account (12), we derive that

$$
\begin{aligned}
\mathcal{G}_{2}(N)= & \sum_{n_{1}+n_{2}=N} \Lambda\left(n_{1}\right) \Lambda\left(n_{2}\right) \Psi_{K}\left(\gamma n_{1}+\delta\right) \Psi_{K}\left(\gamma n_{2}+\delta\right) \\
& +O\left(\left(\Delta+K^{-1} \Delta^{-1}+N^{-1 /(2 \tau)}\right) N(\log N)^{2}\right) .
\end{aligned}
$$

For a given real number $Z \geq 2$, we now split $\Lambda(n)$ as follows:

$$
\Lambda(n)=-\sum_{d \mid n} \mu(d) \log d=\Lambda^{\sharp}(n)+\Lambda^{b}(n),
$$

where

$$
\Lambda^{\sharp}(n)=-\sum_{\substack{d \mid n \\ d \leq Z}} \mu(d) \log d \quad \text { and } \quad \Lambda^{b}(n)=-\sum_{\substack{d \mid n \\ d>Z}} \mu(d) \log d .
$$

Then

$$
\begin{aligned}
\mathcal{G}_{2}(N)= & \mathcal{G}_{2}^{\sharp \sharp}(N)+2 \mathcal{G}_{2}^{\sharp b}(N)+\mathcal{G}_{2}^{\text {bb }}(N) \\
& +O\left(\left(\Delta+K^{-1} \Delta^{-1}+N^{-1 /(2 \tau)}\right) N(\log N)^{2}\right),
\end{aligned}
$$


where

$$
\begin{aligned}
& \mathcal{G}_{2}^{\sharp \sharp}(N)=\sum_{n_{1}+n_{2}=N} \Lambda^{\sharp}\left(n_{1}\right) \Lambda^{\sharp}\left(n_{2}\right) \Psi_{K}\left(\gamma n_{1}+\delta\right) \Psi_{K}\left(\gamma n_{2}+\delta\right), \\
& \mathcal{G}_{2}^{\sharp b}(N)=\sum_{n_{1}+n_{2}=N} \Lambda^{\sharp}\left(n_{1}\right) \Lambda^{b}\left(n_{2}\right) \Psi_{K}\left(\gamma n_{1}+\delta\right) \Psi_{K}\left(\gamma n_{2}+\delta\right), \\
& \mathcal{G}_{2}^{b b}(N)=\sum_{n_{1}+n_{2}=N} \Lambda^{b}\left(n_{1}\right) \Lambda^{b}\left(n_{2}\right) \Psi_{K}\left(\gamma n_{1}+\delta\right) \Psi_{K}\left(\gamma n_{2}+\delta\right) .
\end{aligned}
$$

From now on, let $X$ be a large integer, and put

$$
\Delta=X^{-1 /(8 \tau)} \text { and } K=X^{1 /(4 \tau)} .
$$

Then for all $N \leq X$ the estimate (17) implies

$$
\mathcal{G}_{2}(N)=\mathcal{G}_{2}^{\sharp \sharp}(N)+2 \mathcal{G}_{2}^{\sharp b}(N)+\mathcal{G}_{2}^{\text {bb }}(N)+O\left(X^{1-1 /(10 \tau)}\right) .
$$

Therefore, for any complex numbers $c_{N}$, it follows that

$$
\begin{aligned}
\sum_{N \leq X} c_{N} \mathcal{G}_{2}(N)= & \sum_{N \leq X} c_{N}\left(\mathcal{G}_{2}^{\sharp \sharp}(N)+2 \mathcal{G}_{2}^{\sharp b}(N)+\mathcal{G}_{2}^{b b}(N)\right) \\
& +O\left(\|c\|_{2} X^{3 / 2-1 /(10 \tau)}\right) .
\end{aligned}
$$

Next, we need the following result, the proof of which is given below:

Lemma 5. For any complex numbers $u_{l}$ and $v_{m}$, the bound

$$
\sum_{l+m+n=X} u_{l} v_{m} \Lambda^{b}(n) \Psi_{K}(\gamma n+\delta) \ll\|u\|_{2}\|v\|_{2} \frac{X(\log X)^{2}}{(\log Z)^{A}}
$$

holds with any $A>0$, where

$$
\|u\|_{2}=\left(\sum_{l \leq X}\left|u_{l}\right|^{2}\right)^{1 / 2}, \quad\|v\|_{2}=\left(\sum_{m \leq X}\left|v_{m}\right|^{2}\right)^{1 / 2},
$$

and the implied constant depends only on $\alpha$ and $A$.

For any complex numbers $c_{N}$, we have

$$
\sum_{N \leq X} c_{N} \mathcal{G}_{2}^{\sharp b}(N)=\sum_{l+m+n=X} c_{X-l} \Lambda^{\sharp}(m) \Psi_{K}(\gamma m+\delta) \cdot \Lambda^{b}(n) \Psi_{K}(\gamma n+\delta) .
$$

We now apply Lemma 5 with

$$
\begin{aligned}
u_{l} & = \begin{cases}c_{X-l} & \text { if } 1 \leq l \leq X, \\
0 & \text { otherwise, }\end{cases} \\
v_{m} & = \begin{cases}\Lambda^{\sharp}(m) \Psi_{K}(\gamma m+\delta) & \text { if } 1 \leq m \leq X, \\
0 & \text { otherwise. }\end{cases}
\end{aligned}
$$

From the trivial bound

$$
\left|\Lambda^{\sharp}(m) \Psi_{K}(\gamma m+\delta)\right| \leq d(m) \log m,
$$


where $d(m)$ is the number of positive integer divisors of $m$, it follows that

$$
\|v\|_{2}^{2} \ll X(\log X)^{5}
$$

where we have used the well known bound $\sum_{m \leq X} d(m)^{2} \ll X(\log X)^{3}$ (see, for example, the proof given by Hua [4, Theorem 5.3]; see also [12, 17, 18]). Hence, using Lemma 5 with $A=C+9 / 2$ we derive the bound

$$
\sum_{N \leq X} c_{N} \mathcal{G}_{2}^{\sharp b}(N) \ll\|c\|_{2} \frac{X^{3 / 2}(\log X)^{9 / 2}}{(\log Z)^{C+9 / 2}}
$$

for any constant $C>0$. Similarly,

$$
\sum_{N \leq X} c_{N} \mathcal{G}_{2}^{b b}(N) \ll\|c\|_{2} \frac{X^{3 / 2}(\log X)^{9 / 2}}{(\log Z)^{C+9 / 2}}
$$

Turning to the sum $\mathcal{G}_{2}^{\sharp \sharp}(N)$, we begin by inserting the Fourier expansion of $\Psi_{K}(x)$ and then changing the order of summation, obtaining

$$
\begin{aligned}
\mathcal{G}_{2}^{\sharp \sharp}(N) & =\sum_{n_{1}+n_{2}=N} \Lambda^{\sharp}\left(n_{1}\right) \Lambda^{\sharp}\left(n_{2}\right) \Psi_{K}\left(\gamma n_{1}+\delta\right) \Psi_{K}\left(\gamma n_{2}+\delta\right) \\
& =\sum_{n \leq N} \Lambda^{\sharp}(n) \Lambda^{\sharp}(N-n) \Psi_{K}(\gamma n+\delta) \Psi_{K}(\gamma(N-n)+\delta) \\
& =\sum_{\substack{|k| \leq K \\
|l| \leq K}} g(k) g(l) \mathbf{e}(k \delta) \mathbf{e}(l(\gamma N+\delta)) \sum_{n \leq N} \Lambda^{\sharp}(n) \Lambda^{\sharp}(N-n) \mathbf{e}((k-l) \gamma n) .
\end{aligned}
$$

We now collect terms in the double sum according to whether $k=l$ or not. Writing

$$
G_{2}^{\sharp \sharp}(N)=\sum_{n \leq N} \Lambda^{\sharp}(n) \Lambda^{\sharp}(N-n),
$$

the contribution to $\mathcal{G}_{2}^{\sharp}(N)$ coming from terms with $k=l$ is

$$
G_{2}^{\sharp \sharp}(N) \sum_{|k| \leq K} g(k)^{2} \mathbf{e}(k(\gamma N+2 \delta))=\Psi_{K}^{(2)}(\gamma N+2 \delta) G_{2}^{\sharp \sharp}(N),
$$

where we have used (16) in the second step. To bound the remainder

$$
R=\sum_{\substack{|k|,|l| \leq K \\ k \neq l}} g(k) g(l) \mathbf{e}(k \delta) \mathbf{e}(l(\gamma N+\delta)) \sum_{n \leq N} \Lambda^{\sharp}(n) \Lambda^{\sharp}(N-n) \mathbf{e}((k-l) \gamma n),
$$

we use the following result, the proof of which is given below:

LEMMA 6. For every integer $k_{0} \neq 0$ with $\left|k_{0}\right| \leq 2 K=2 X^{1 /(4 \tau)}$, we have

$$
\sum_{n \leq N} \Lambda^{\sharp}(n) \Lambda^{\sharp}(N-n) \mathbf{e}\left(k_{0} \gamma n\right) \ll X^{1 / 2} Z^{3+4 \tau},
$$

where the implied constant depends only on $\alpha$. 
From Lemma 6, it follows that

$$
R \ll X^{1 / 2} Z^{3+4 \tau} \sum_{|k| \leq K}|g(k)| \sum_{|l| \leq K}|g(l)| \ll X^{1 / 2} Z^{3+4 \tau}(\log X)^{2},
$$

where we have used (9) together with our choice of $K$.

We have therefore shown that

$$
\mathcal{G}_{2}^{\sharp \sharp}(N)=\Psi_{K}^{(2)}(\gamma N+2 \delta) G_{2}^{\sharp \sharp}(N)+O\left(X^{1 / 2} Z^{3+4 \tau}(\log X)^{2}\right) .
$$

For any complex numbers $c_{N}$, it follows that

$$
\sum_{N \leq X} c_{N} \mathcal{G}_{2}^{\sharp \sharp}(N)=\sum_{N \leq X} c_{N} \Psi_{K}^{(2)}(\gamma N+2 \delta) G_{2}^{\sharp \sharp}(N)+O\left(\|c\|_{2} X Z^{3+4 \tau}(\log X)^{2}\right) .
$$

Now put $Z=X^{1 /(9+12 \tau)}$. Using the previous estimate together with the bounds (20) and (21), we derive from (19) the estimate

$$
\sum_{N \leq X} c_{N} \mathcal{G}_{2}(N)=\sum_{N \leq X} c_{N} \Psi_{K}^{(2)}(\gamma N+2 \delta) G_{2}^{\sharp \sharp}(N)+O\left(\|c\|_{2} \frac{X^{3 / 2}}{(\log X)^{C}}\right) .
$$

Examining the proof of [6, Lemma 19.3] (which is stated only for even numbers $N$ but holds for odd numbers as well) and taking into account the identity (4) with $\kappa=2$, we deduce that

$$
G_{2}^{\sharp \sharp}(N)=\mathfrak{S}_{2}(N) N+O\left(\frac{N}{(\log N)^{C}}\right) .
$$

From the trivial estimate

$$
\sum_{N \leq X} c_{N} \Psi_{K}^{(2)}(\gamma N+2 \delta) \ll\|c\|_{2} X^{1 / 2}
$$

it follows that

$$
\sum_{N \leq X} c_{N} \mathcal{G}_{2}(N)=\sum_{N \leq X} c_{N} \Psi_{K}^{(2)}(\gamma N+2 \delta) \mathfrak{S}_{2}(N) N+O\left(\|c\|_{2} \frac{X^{3 / 2}}{(\log X)^{C}}\right) .
$$

Finally, by (15) and our choices of $\Delta$ and $K$, we have

$$
\Psi_{K}^{(2)}(x)=\psi^{(2)}(x)+O\left(X^{-1 /(8 \tau)}\right) .
$$

In view of the trivial bound (6), it follows that

$$
X^{-1 /(8 \tau)} \sum_{N \leq X} c_{N} \mathfrak{S}_{2}(N) N \ll\|c\|_{2} X^{3 / 2-1 /(8 \tau)} \log \log X ;
$$

therefore,

$$
\sum_{N \leq X} c_{N} \mathcal{G}_{2}(N)=\sum_{N \leq X} c_{N} \psi^{(2)}(\gamma N+2 \delta) \mathfrak{S}_{2}(N) N+O\left(\|c\|_{2} \frac{X^{3 / 2}}{(\log X)^{C}}\right)
$$

as required. 
Proof of Lemma 5. We argue as in [6, Section 19.3] and begin with a bound for the exponential sum

$$
S_{\Psi_{K}}^{b}(\xi)=\sum_{n \leq X} \Lambda^{b}(n) \Psi_{K}(\gamma n+\beta) \mathbf{e}(\xi n) .
$$

From the definition (13), it follows that

$$
\left|S_{\Psi_{K}}^{b}(\xi)\right| \leq \sum_{|k| \leq K}\left|g(k) S^{b}(\xi+k \gamma)\right|, \quad \text { where } \quad S^{b}(\xi)=\sum_{n \leq X} \Lambda^{b}(n) \mathbf{e}(\xi n) .
$$

Using the bound (19.17) from [6] together with (9), we immediately deduce that the uniform bound

$$
\left|S_{\Psi_{K}}^{b}(\xi)\right| \ll \frac{X \log X \log K}{(\log Z)^{A}} \quad(\xi \in \mathbb{R})
$$

holds with any fixed constant $A>0$.

To complete the proof, we observe that

$$
\begin{aligned}
& \sum_{l+m+n=X} u_{l} v_{m} \Lambda^{b}(n) \Psi_{K}(\gamma n+\delta) \\
&=\int_{0}^{1}\left(\sum_{l \leq X} u_{l} \mathbf{e}(\xi l)\right)\left(\sum_{m \leq X} v_{m} \mathbf{e}(\xi m)\right) S_{\Psi_{K}}^{b}(\xi) \mathbf{e}(-\xi X) d \xi .
\end{aligned}
$$

Applying the Cauchy-Schwarz inequality and using (22) (with $K=X^{1 /(4 \tau)}$ ) together with the equalities

$$
\int_{0}^{1}\left|\sum_{l \leq X} u_{l} \mathbf{e}(\xi l)\right|^{2} d \xi=\sum_{l \leq X}\left|u_{l}\right|^{2}, \quad \int_{0}^{1}\left|\sum_{m \leq X} v_{m} \mathbf{e}(\xi m)\right|^{2} d \xi=\sum_{m \leq X}\left|v_{m}\right|^{2},
$$

we obtain the stated bound.

Proof of Lemma 6. We have:

$$
\begin{aligned}
\sum_{n \leq N} \Lambda^{\sharp}(n) & \Lambda^{\sharp}(N-n) \mathbf{e}\left(k_{0} \gamma n\right) \\
= & \sum_{n \leq N}\left(\sum_{\substack{d_{1} \mid n \\
d_{1} \leq Z}} \mu\left(d_{1}\right) \log d_{1}\right)\left(\sum_{\substack{d_{2} \mid N-n \\
d_{2} \leq Z}} \mu\left(d_{2}\right) \log d_{2}\right) \mathbf{e}\left(k_{0} \gamma n\right) \\
= & \sum_{d_{1}, d_{2} \leq Z} \mu\left(d_{1}\right) \mu\left(d_{2}\right) \log d_{1} \log d_{2} \sum_{\substack{l_{1}, l_{2} \geq 1 \\
l_{1} d_{1}+l_{2} d_{2}=N}} \mathbf{e}\left(k_{0} \gamma l_{1} d_{1}\right) .
\end{aligned}
$$

If $l_{1} \geq 1$, then $l_{1} d_{1}+l_{2} d_{2}=N$ for some $l_{2} \geq 1$ if and only if $l_{1}<N / d_{1}$, $f=\operatorname{gcd}\left(d_{1}, d_{2}\right)$ is a divisor of $N$, and

$$
l_{1}\left(d_{1} / f\right) \equiv(N / f)\left(\bmod d_{2} / f\right) .
$$


Let $a$ be the least positive integer such that

$$
a \equiv\left(d_{1} / f\right)^{-1}(N / f)\left(\bmod d_{2} / f\right) .
$$

Then $l_{1}$ varies over the set $\left\{a, a+d_{2} / f, \ldots, a+(L-1) d_{2} / f\right\}$, where

$$
L=\left\lfloor\frac{N / d_{1}-a}{d_{2} / f}\right\rfloor=\frac{N}{\left[d_{1}, d_{2}\right]}+O(1),
$$

and it follows that

$$
\begin{aligned}
\sum_{\substack{l_{1}, l_{2} \geq 1 \\
l_{1} d_{1}+l_{2} d_{2}=N}} \mathbf{e}\left(k_{0} \gamma l_{1} d_{1}\right) & =\mathbf{e}\left(k_{0} \gamma a d_{1}\right) \sum_{j=0}^{L-1} \mathbf{e}\left(k_{0} \gamma j\left[d_{1}, d_{2}\right]\right) \\
& \ll \frac{1}{\llbracket k_{0} \gamma\left[d_{1}, d_{2}\right] \rrbracket},
\end{aligned}
$$

where we have used a standard estimate in the second step (see, for example, [8, Chapter 1, Lemma 1]). Since $\gamma$ is of type $\tau$, we have

$$
\llbracket \gamma n \rrbracket \gg n^{-2 \tau} \quad(n \geq 1),
$$

where the implied constant depends on $\alpha$; thus,

$$
\frac{1}{\llbracket k_{0} \gamma\left[d_{1}, d_{2}\right] \rrbracket} \ll k_{0}^{2 \tau}\left[d_{1}, d_{2}\right]^{2 \tau} \leq\left(2 X^{1 /(4 \tau)}\right)^{2 \tau} Z^{4 \tau} \ll X^{1 / 2} Z^{4 \tau} .
$$

Combining this bound with (23) and (24), and using the trivial bound

$$
\sum_{d_{1}, d_{2} \leq Z} \log d_{1} \log d_{2} \leq Z^{2}(\log Z)^{2} \ll Z^{3},
$$

we obtain the desired result.

4. Three or more Beatty primes. In what follows, we use the same notation as in the proof of Theorem 4, except that we now define

$$
\Delta=N^{-1 /(8 \tau)}, \quad K=N^{1 /(4 \tau)}
$$

instead of (18). With these choices, we have the following analog of (15) for every $\kappa \geq 2$ :

$$
\Psi_{K}^{(\kappa)}(x)=\psi^{(\kappa)}(x)+O\left(N^{-1 /(8 \tau)}\right) \quad(x \in \mathbb{R}) .
$$

Also,

$$
\Psi_{K}^{(\kappa)}(x)=\sum_{|l| \leq K} g(l)^{\kappa} \mathbf{e}(l x) .
$$

Proposition 1. Let $\kappa \geq 2$ be fixed. If, for any constant $C>0$, the estimate

$$
\mathcal{G}_{\kappa}(n)=\Psi_{K}^{(\kappa)}(\gamma n+\kappa \delta) \mathfrak{S}_{\kappa}(n) \frac{n^{\kappa-1}}{(\kappa-1) !}+O\left(\frac{n^{\kappa-1}}{(\log n)^{C}}\right)
$$


holds for all but $O\left(N(\log N)^{-C}\right)$ integers $n \leq N$, then the estimate

$$
\mathcal{G}_{\kappa+1}(N)=\Psi_{K}^{(\kappa+1)}(\gamma N+(\kappa+1) \delta) \mathfrak{S}_{\kappa+1}(N) \frac{N^{\kappa}}{\kappa !}+O\left(\frac{N^{\kappa}}{(\log N)^{C}}\right)
$$

holds with any constant $C>0$.

REMARK. This result immediately yields a proof of Theorem 3. Indeed, using (6) and (25) we obtain (27) with $\kappa=2$. By induction, Proposition 1 implies that (28) holds for every fixed $\kappa \geq 2$. Replacing $\kappa$ by $\kappa-1$ in (28) and then using the estimate (25) again, we obtain the statement of Theorem 3.

Proof of Proposition 1. To simplify our exposition in what follows, for any functions $F=F(N)$ and $G=G(N)$ we use the notation

$$
F=\widetilde{O}(G)
$$

to mean that for any choice of the constant $C>0$ the inequality

$$
|F| \leq c \frac{|G|}{(\log N)^{C}}
$$

holds for all $N \geq 2$ with a constant $c>0$ that depends only on $\alpha, \kappa$ and $C$.

By Lemma 2 and the definition (2), we have

$$
\begin{aligned}
\mathcal{G}_{\kappa+1}(N) & =\sum_{n_{1}+\cdots+n_{\kappa+1}=N} \Lambda\left(n_{1}\right) \cdots \Lambda\left(n_{\kappa+1}\right) \psi\left(\gamma n_{1}+\delta\right) \cdots \psi\left(\gamma n_{\kappa+1}+\delta\right) \\
& =\sum_{n \leq N} \Lambda(N-n) \psi(\gamma(N-n)+\delta) \mathcal{G}_{\kappa}(n) \\
& =\sum_{n \leq N}^{*} \Lambda(N-n) \psi(\gamma(N-n)+\delta) \mathcal{G}_{\kappa}(n)+\widetilde{O}\left(N^{\kappa}\right),
\end{aligned}
$$

where $\sum^{*}$ indicates that the sum is restricted to integers $n$ satisfying (27); note that we have used the trivial bound

$$
\Lambda(N-n) \psi(\gamma(N-n)+\delta) \mathcal{G}_{\kappa}(n) \ll N^{\kappa-1}(\log N)^{\kappa}
$$

to estimate the contribution from exceptional integers. By (27), the previous sum is equal to

$$
\sum_{n \leq N}^{*} \Lambda(N-n) \psi(\gamma(N-n)+\delta) \Psi_{K}^{(\kappa)}(\gamma n+\kappa \delta) \mathfrak{S}_{\kappa}(n) \frac{n^{\kappa-1}}{(\kappa-1) !}+\widetilde{O}\left(N^{\kappa}\right) .
$$

We now extend the sum to all integers $n \leq N$, using (6) or (7) to bound $\mathfrak{S}_{\kappa}(n)$ for each exceptional $n$, then we replace $\psi$ with $\Psi_{K}$ using (25) to control the error term. Finally, replacing $n$ by $N-n$, we see that $\mathcal{G}_{\kappa+1}(N)$ is equal to

$$
\sum_{n \leq N} \Lambda(n) \Psi_{K}(\gamma n+\delta) \Psi_{K}^{(\kappa)}(\gamma(N-n)+\kappa \delta) \mathfrak{S}_{\kappa}(N-n) \frac{(N-n)^{\kappa-1}}{(\kappa-1) !}+\widetilde{O}\left(N^{\kappa}\right)
$$


In this sum, we substitute the Fourier expansions (13) and (26) for $\Psi_{K}$ and $\Psi_{K}^{(\kappa)}$, respectively, then change the order of summation, obtaining

$$
\mathcal{G}_{\kappa+1}(N)=\sum_{|k|,|l| \leq K} g(k) g(l)^{\kappa} \mathbf{e}(k \delta+l \gamma N+l \kappa \delta) \frac{S_{k, l}(N)}{(\kappa-1) !}+\widetilde{O}\left(N^{\kappa}\right),
$$

where

$$
S_{k, l}(N)=\sum_{n \leq N} \Lambda(n) \mathbf{e}((k-l) \gamma n) \mathfrak{S}_{\kappa}(N-n)(N-n)^{\kappa-1} .
$$

We now show that the main contribution to $\mathcal{G}_{\kappa+1}(N)$ comes from the sums $S_{k, l}(N)$ with $k=l$. To this end, we use (4) to write

$$
\begin{aligned}
S_{k, l}(N) & =\sum_{n \leq N} \Lambda(n) \mathbf{e}((k-l) \gamma n)(N-n)^{\kappa-1} \sum_{d \mid N-n} \sum_{\substack{c \geq 1 \\
\operatorname{gcd}(c, d)=1}} \frac{\mu(c)^{\kappa+1} \mu(d)^{\kappa} d}{\varphi(c)^{\kappa} \varphi(d)^{\kappa}} \\
& =\sum_{d \leq N} \sum_{\substack{c \geq 1 \\
\operatorname{gcd}(c, d)=1}} \frac{\mu(c)^{\kappa+1} \mu(d)^{\kappa} d}{\varphi(c)^{\kappa} \varphi(d)^{\kappa}} T_{k, l, d}(N),
\end{aligned}
$$

where

$$
T_{k, l, d}(N)=\sum_{\substack{n \leq N \\ n \equiv N(\bmod d)}} \Lambda(n) \mathbf{e}((k-l) \gamma n)(N-n)^{\kappa-1} .
$$

Using the trivial uniform bound

$$
T_{k, l, d}(N) \ll \frac{N^{\kappa} \log N}{d}
$$

and the well known lower bound $\varphi(d) \gg d / \log \log d$, for any $y>3$ we have (since $\kappa \geq 2$ )

$$
\begin{aligned}
\sum_{d>y} \sum_{\substack{c \geq 1 \\
\operatorname{gcd}(c, d)=1}} \frac{\mu(c)^{\kappa+1} \mu(d)^{\kappa} d}{\varphi(c)^{\kappa} \varphi(d)^{\kappa}} T_{k, l, d}(N) & \ll \sum_{d>y} \frac{d(\log \log d)^{\kappa}}{d^{\kappa}} \frac{N^{\kappa} \log N}{d} \\
& \ll N^{\kappa} \log N \sum_{d>y} \frac{1}{d^{3 / 2}} \ll \frac{N^{\kappa} \log N}{y^{1 / 2}} .
\end{aligned}
$$

Taking $y=(\log N)^{A}$ with $A=2 C+2$ and $C>0$ arbitrary, we derive that

$$
S_{k, l}(N)=\sum_{d \leq(\log N)^{A}} \sum_{\substack{c \geq 1 \\ \operatorname{gcd}(c, d)=1}} \frac{\mu(c)^{\kappa+1} \mu(d)^{\kappa} d}{\varphi(c)^{\kappa} \varphi(d)^{\kappa}} T_{k, l, d}(N)+O\left(\frac{N^{\kappa}}{(\log N)^{C}}\right) .
$$

Next, we observe that if $d \leq(\log N)^{A}$ and $\operatorname{gcd}(d, N) \neq 1$, then the number $\omega(d)$ of distinct prime divisors of $d$ satisfies the bound $\omega(d) \ll \log \log N$, 
and it is easy to see that the bound

$$
T_{k, l, d}(N) \ll N^{\kappa-1} \log N \log \log N
$$

holds for all such $d$. Using this estimate in the preceding expression for $S_{k, l}(N)$, we deduce that

$$
S_{k, l}(N)=\sum_{\substack{d \leq(\log N)^{A} \\ \operatorname{gcd}(d, N)=1}} \sum_{\substack{c \geq 1 \\ \operatorname{gcd}(c, d)=1}} \frac{\mu(c)^{\kappa+1} \mu(d)^{\kappa} d}{\varphi(c)^{\kappa} \varphi(d)^{\kappa}} T_{k, l, d}(N)+O\left(\frac{N^{\kappa}}{(\log N)^{C}}\right) .
$$

In the case where $k=l$, Lemma 3 immediately implies that

$$
T_{k, k, d}(N)=\sum_{\substack{n \leq N \\ n \equiv N(\bmod d)}} \Lambda(n)(N-n)^{\kappa-1}=\frac{N^{\kappa}}{\kappa \varphi(d)}+\widetilde{O}\left(N^{\kappa}\right)
$$

and therefore,

$$
S_{k, k}(N)=\frac{N^{\kappa}}{\kappa} \sum_{\substack{d \leq(\log N)^{A} \\ \operatorname{gcd}(d, N)=1}} \sum_{\substack{c \geq 1 \\ \operatorname{gcd}(c, d)=1}} \frac{\mu(c)^{\kappa+1} \mu(d)^{\kappa} d}{\varphi(c)^{\kappa} \varphi(d)^{\kappa+1}}+O\left(\frac{N^{\kappa}}{(\log N)^{C}}\right) .
$$

Since

$$
\sum_{\substack{d>(\log N)^{A} \\ \operatorname{gcd}(d, N)=1}} \sum_{\substack{c \geq 1 \\ \operatorname{gcd}(c, d)=1}} \frac{\mu(c)^{\kappa+1} \mu(d)^{\kappa} d}{\varphi(c)^{\kappa} \varphi(d)^{\kappa+1}} \ll \sum_{d>(\log N)^{A}} \frac{(\log \log d)^{\kappa+1}}{d^{\kappa}} \ll \frac{1}{(\log N)^{C+1}},
$$

and $C$ is arbitrary, it follows that

$$
S_{k, k}(N)=\frac{N^{\kappa}}{\kappa} \sum_{\substack{c, d \geq 1 \\ \operatorname{gcd}(d, c N)=1}} \frac{\mu(c)^{\kappa+1} \mu(d)^{\kappa} d}{\varphi(c)^{\kappa} \varphi(d)^{\kappa+1}}+\widetilde{O}\left(N^{\kappa}\right) .
$$

Finally, using (5) (with $\kappa$ replaced by $\kappa+1$ ) we deduce that

$$
S_{k, k}(N)=\mathfrak{S}_{\kappa+1}(N) \frac{N^{\kappa}}{\kappa}+\widetilde{O}\left(N^{\kappa}\right) \quad(|k| \leq K) .
$$

To treat the case $k \neq l$, we use the following result, the proof of which is given below:

LEMMA 7. There exists a constant $\eta>0$ that depends only on $\alpha$ and has the following property. For any positive integer $d$ coprime to $N$, and any non-zero integer $k_{0}$ such that $\left|k_{0}\right| \leq 2 N^{1 /(4 \tau)}$,

$$
\sum_{\substack{n \leq N \\ n \equiv N(\bmod d)}} \Lambda(n) \mathbf{e}\left(k_{0} \gamma n\right)(N-n)^{\kappa-1} \ll N^{\kappa-\eta},
$$

where the implied constant depends only on $\kappa$. 
By Lemma 7 we have, for all $|k|,|l| \leq K$ with $k \neq l$,

$$
T_{k, l, d}(N)=\sum_{\substack{n \leq N \\ n \equiv N(\bmod d)}} \Lambda(n) \mathbf{e}((k-l) \gamma n)(N-n)^{\kappa-1}=\widetilde{O}\left(N^{\kappa}\right),
$$

and therefore,

$$
S_{k, l}(N)=\widetilde{O}\left(N^{\kappa}\right) \quad(|k|,|l| \leq K, k \neq l) .
$$

Inserting the estimates (30) and (31) into (29), and taking into account (9), leads to

$$
\begin{aligned}
\mathcal{G}_{\kappa+1}(N) & =\mathfrak{S}_{\kappa+1}(N) \frac{N^{\kappa}}{\kappa !} \sum_{|k| \leq K} g(k)^{\kappa+1} \mathbf{e}(k \gamma N+(\kappa+1) k \delta)+\widetilde{O}\left(N^{\kappa}\right) \\
& =\Psi_{K}^{(\kappa+1)}(\gamma N+(\kappa+1) \delta) \mathfrak{S}_{\kappa+1}(N) \frac{N^{\kappa}}{\kappa !}+\widetilde{O}\left(N^{\kappa}\right),
\end{aligned}
$$

and this completes the proof.

Proof of Lemma \%. Fix a constant $\varrho$ such that

$$
1 \leq \tau<\varrho<2 \tau .
$$

Since $\gamma$ is of type $\tau$, for some constant $c_{0}>0$ we have

$$
\llbracket \gamma m \rrbracket>c_{0} m^{-\varrho} \quad(m \geq 1) .
$$

Taking $c_{0}$ smaller if necessary, we can assume that $c_{0}<2^{\varrho}$. Put

$$
c_{1}=2^{\varrho} / c_{0} \text { and } \varepsilon=1 /(4 \tau+2) .
$$

Let $d$ and $k_{0}$ be integers with the properties stated in the lemma; without loss of generality, we can assume that $k_{0}$ is positive. Let $a / b$ be the convergent in the continued fraction expansion of $k_{0} \gamma$ that has the largest denominator $b$ not exceeding $c_{1} N^{1-\varepsilon}$; then

$$
\left|k_{0} \gamma-\frac{a}{b}\right| \leq \frac{1}{b c_{1} N^{1-\varepsilon}}=\frac{c_{0}}{b 2^{\varrho} N^{1-\varepsilon}} .
$$

Multiplying by $b$ and taking (32) into account, we have

$$
\frac{c_{0}}{2^{\varrho} N^{1-\varepsilon}} \geq\left|b k_{0} \gamma-a\right| \geq \llbracket b k_{0} \gamma \rrbracket>c_{0}\left(b k_{0}\right)^{-\varrho} .
$$

Thus, since $k_{0} \leq 2 N^{1 /(4 \tau)}$ and $\varrho<2 \tau$, it follows that

$$
b \geq N^{(1-\varepsilon) /(2 \tau)-1 /(4 \tau)}=N^{\varepsilon} .
$$

Inserting (34) into (33) and recalling that $c_{0}<2^{\varrho}$, we conclude that

$$
\left|k_{0} \gamma-\frac{a}{b}\right| \leq \frac{1}{N}
$$

We are therefore in a position to apply Lemma 4 with $\theta=k_{0} \gamma$, and this yields the stated result immediately since $N^{\varepsilon} \leq b \leq c_{1} N^{1-\varepsilon}$. 
5. Convolutions with $\psi$. In this section, we focus on properties of the $\kappa$-fold convolutions of $\psi$. We recall that $\psi$ is the periodic function with period one defined by

$$
\psi(x)= \begin{cases}1 & \text { if } 0<\{x\} \leq \gamma, \\ 0 & \text { if } \gamma<\{x\}<1 \text { or } x \in \mathbb{Z} .\end{cases}
$$

We assume that $\gamma=\alpha^{-1}<1$. As before, we put $\psi^{(1)}=\psi$, and for every $\kappa \geq 2$, we denote by $\psi^{(\kappa)}$ the $\kappa$-fold convolution of $\psi$ with itself:

$$
\psi^{(\kappa)}(x)=\int_{0}^{1} \psi^{(\kappa-1)}(x-y) \psi(y) d y=\int_{x-\gamma}^{x} \psi^{(\kappa-1)}(y) d y .
$$

Since $0 \leq \psi(x) \leq \gamma$ for all $x \in \mathbb{R}$, it is easy to see that

$$
0 \leq \psi^{(\kappa)}(x) \leq \gamma^{\kappa-1} \quad(\kappa \geq 1, x \in \mathbb{R}) .
$$

Note that $\psi^{(\kappa)}$ is continuous for $\kappa \geq 2$ and differentiable for $\kappa \geq 3$.

Proposition 2. If $\kappa \geq\lceil\alpha\rceil$, then there exists a constant $c>0$ which depends only on $\alpha$ and $\kappa$ such that $\psi^{(\kappa)}(x) \geq c$ for all $x \in \mathbb{R}$.

Proof. By periodicity, it suffices to prove this for all $x$ in $[\varepsilon, 1+\varepsilon]$ for some $\varepsilon>0$. Since $\kappa \gamma \geq\lceil\alpha\rceil / \alpha>1$, there exists $\varepsilon>0$ such that $1+2 \varepsilon \leq \kappa \gamma$. Fixing $\varepsilon$, it is easy to see that for every $x \in[\varepsilon, 1+\varepsilon]$ the closed intervals

$$
\mathcal{I}_{x}=\left[\frac{x}{\kappa}-\frac{\varepsilon}{\kappa}, \frac{x}{\kappa}+\frac{\varepsilon}{\kappa}\right] \quad \text { and } \quad \mathcal{J}_{x}=\left[\frac{x}{\kappa}-\frac{\varepsilon}{\kappa(\kappa-1)}, \frac{x}{\kappa}+\frac{\varepsilon}{\kappa(\kappa-1)}\right]
$$

are contained in $[0, \gamma]$. Also, if $y_{j} \in \mathcal{J}_{x}$ for $j=1, \ldots, \kappa-1$, then the number $x-y_{1}-\cdots-y_{\kappa-1}$ lies in $\mathcal{I}_{x}$. Therefore,

$$
\begin{aligned}
\psi^{(\kappa)}(x) & =\int_{0}^{1} \cdots \int_{0}^{1} \psi\left(y_{1}\right) \cdots \psi\left(y_{\kappa-1}\right) \psi\left(x-y_{1}-\cdots-y_{\kappa-1}\right) d y_{1} \cdots d y_{\kappa-1} \\
& \geq \int_{\mathcal{J}_{x}} \cdots \int_{\mathcal{J}_{x}} d y_{1} \cdots d y_{\kappa-1}=\left(\frac{2 \varepsilon}{\kappa(\kappa-1)}\right)^{\kappa-1}
\end{aligned}
$$

for all $x \in[\varepsilon, 1+\varepsilon]$.

The remainder of this section is devoted to the problem of finding a sharp lower bound for $\psi^{(\kappa)}(x)$ in the special case that $\kappa=\lceil\alpha\rceil$, which is given in Theorem 5 below.

Lemma 8. If $\kappa \geq 2$, then $\psi^{(\kappa)}(x)=\psi^{(\kappa)}(\kappa \gamma-x)$ for all $x \in \mathbb{R}$.

Proof. Let $\psi_{0}$ be the characteristic function of the set of real numbers $x$ such that $\llbracket x \rrbracket \leq \gamma / 2$. Clearly, $\psi(x)=\psi_{0}(x-\gamma / 2)$ for all $x \in \mathbb{R} \backslash \mathbb{Z}$, and by induction on $\kappa$, we have $\psi^{(\kappa)}(x)=\psi_{0}^{(\kappa)}(x-\kappa \gamma / 2)$ for all $\kappa \geq 2$ and $x \in \mathbb{R}$. 
Since $\psi_{0}$ is an even function, so is $\psi_{0}^{(\kappa)}$ for all $\kappa \geq 2$; therefore,

$$
\psi^{(\kappa)}(x)=\psi_{0}^{(\kappa)}(x-\kappa \gamma / 2)=\psi_{0}^{(\kappa)}(\kappa \gamma / 2-x)=\psi^{(\kappa)}(\kappa \gamma-x)
$$

for all $\kappa \geq 2$ and $x \in \mathbb{R}$.

Lemma 9. If $1 \leq \kappa<\lceil\alpha\rceil$ and $x \in(\kappa \gamma, 1]$, then $\psi^{(\kappa)}(x)=0$.

Proof. When $\kappa=1$, this follows from the definition of $\psi$. Now suppose that $\psi^{(\kappa-1)}(x)=0$ for all $x \in((\kappa-1) \gamma, 1]$, where $\kappa \geq 2$. Then for each $x \in(\kappa \gamma, 1]$ the interval $[x-\gamma, x]$ is contained in $((\kappa-1) \gamma, 1]$; therefore,

$$
\psi^{(\kappa)}(x)=\int_{x-\gamma}^{x} \psi^{(\kappa-1)}(y) d y=0,
$$

and the result follows by induction.

The next result is an easy consequence of Lemma 9:

LEMMA 10. If $2 \leq \kappa<\lceil\alpha\rceil$ and $x \in[0, \gamma]$, then

$$
\psi^{(\kappa)}(x)=\int_{0}^{x} \psi^{(\kappa-1)}(y) d y .
$$

The same result holds for $\kappa=\lceil\alpha\rceil$ and $x \in[\kappa \gamma-1, \gamma]$.

LEMMA 11. For $1 \leq \kappa<\lceil\alpha\rceil$ and $x \in(0, \gamma]$, we have

$$
\psi^{(\kappa)}(x)=\frac{x^{\kappa-1}}{(\kappa-1) !} .
$$

Proof. This is immediate for $\kappa=1$. Suppose $\psi^{(\kappa-1)}(x)=x^{\kappa-2} /(\kappa-2)$ ! for $x \in(0, \gamma]$, where $2 \leq \kappa<\lceil\alpha\rceil$. Then by Lemma 10 we have

$$
\psi^{(\kappa)}(x)=\int_{0}^{x} \psi^{(\kappa-1)}(y) d y=\int_{0}^{x} \frac{y^{\kappa-2}}{(\kappa-2) !} d y=\frac{x^{\kappa-1}}{(\kappa-1) !},
$$

and the result follows by induction.

LEMMA 12. If $1 \leq \kappa<\lceil\alpha\rceil$, then $\psi^{(\kappa)}$ is increasing on $[0, \kappa \gamma / 2]$.

Proof. For $\kappa=1$ this is immediate, and for $\kappa=2$ it follows from the fact that $\psi^{(2)}(x)=x$ for $x \in[0, \gamma]$ by Lemma 11 and the continuity of $\psi^{(2)}$. Now suppose that $\psi^{(\kappa-1)}$ is increasing on $[0,(\kappa-1) \gamma / 2]$, where $\kappa \geq 3$. Since $\psi^{(\kappa)}$ is differentiable, for $x \in[\gamma,(\kappa-1) \gamma / 2]$ we have

$$
\left.\frac{d \psi^{(\kappa)}(t)}{d t}\right|_{t=x}=\psi^{(\kappa-1)}(x)-\psi^{(\kappa-1)}(x-\gamma) \geq 0 .
$$

If $x \in[0, \gamma]$, then by Lemma 10 it follows that

$$
\left.\frac{d \psi^{(\kappa)}(t)}{d t}\right|_{t=x}=\psi^{(\kappa-1)}(x)-\psi^{(\kappa-1)}(0) \geq 0
$$


Finally, suppose that $x \in[(\kappa-1) \gamma / 2, \kappa \gamma / 2]$. Since $\psi^{(\kappa-1)}$ is increasing on $[0,(\kappa-1) \gamma / 2]$, it is decreasing on $[(\kappa-1) \gamma / 2,(\kappa-1) \gamma]$ by Lemma 8 ; therefore, using the same lemma we have

$$
\begin{aligned}
\left.\frac{d \psi^{(\kappa)}(t)}{d t}\right|_{t=x} & =\psi^{(\kappa-1)}(x)-\psi^{(\kappa-1)}(x-\gamma) \\
& \geq \psi^{(\kappa-1)}(\kappa \gamma / 2)-\psi^{(\kappa-1)}((\kappa-2) \gamma / 2)=0,
\end{aligned}
$$

and the proof is completed by induction.

TheOREM 5. For $\kappa=\lceil\alpha\rceil$, the sharp lower bound

$$
\psi^{(\kappa)}(x) \geq \frac{(\kappa \gamma-1)^{\kappa-1}}{2^{\kappa-2}(\kappa-1) !}
$$

holds uniformly for all $x \in \mathbb{R}$.

Proof. Since $\psi^{(\kappa)}$ has period one, we can assume that $x \in[0,1]$.

Using Lemmas 8 and 10 and arguing as in the proof of Lemma 12, one sees that $\psi^{(\kappa)}$ is increasing on the interval $[\kappa \gamma-1, \kappa \gamma / 2]$ and decreasing on the interval $[\kappa \gamma / 2,1]$. Therefore,

$$
\psi^{(\kappa)}(x) \geq \psi^{(\kappa)}(1)=\psi^{(\kappa)}(0)
$$

for all $x \in[\kappa \gamma-1,1]$. On the other hand, by Lemmas 8, 9 and 11, for $x \in[0, \kappa \gamma-1]$ we have

$$
\begin{aligned}
\psi^{(\kappa)}(x) & =\int_{x+1-\gamma}^{1} \psi^{(\kappa-1)}(y) d y+\int_{0}^{x} \psi^{(\kappa-1)}(y) d y \\
& =\int_{(\kappa-1) \gamma-1}^{\kappa \gamma-1-x} \psi^{(\kappa-1)}(y) d y+\int_{0}^{x} \psi^{(\kappa-1)}(y) d y \\
& =\int_{0}^{\kappa \gamma-1-x} \psi^{(\kappa-1)}(y) d y+\int_{0}^{x} \psi^{(\kappa-1)}(y) d y=f(x),
\end{aligned}
$$

where

$$
f(x)=\frac{(\kappa \gamma-1-x)^{\kappa-1}+x^{\kappa-1}}{(\kappa-1) !} .
$$

Since the function $f(x)$ attains its minimum on $[0, \kappa \gamma-1]$ at $x=(\kappa \gamma-1) / 2$, we obtain the stated result.

6. Proof of Theorem 1. Suppose that $\kappa<\alpha$. If $N \equiv \kappa(\bmod 2)$, and

$$
N=\left\lfloor\alpha m_{1}+\beta\right\rfloor+\left\lfloor\alpha m_{2}+\beta\right\rfloor+\cdots+\left\lfloor\alpha m_{\kappa}+\beta\right\rfloor
$$

for some $m_{1}, \ldots, m_{\kappa} \in \mathbb{N}$, then

$$
(N-\kappa \beta) \alpha^{-1} \leq m_{1}+\cdots+m_{\kappa}<(N-\kappa \beta) \alpha^{-1}+\kappa \alpha^{-1} .
$$


Therefore, the relation (35) cannot hold if the fractional part $\left\{(N-\kappa \beta) \alpha^{-1}\right\}$ of $(N-\kappa \beta) \alpha^{-1}$ lies in the open interval $\left(0,1-\kappa \alpha^{-1}\right)$, which happens for about $\frac{1}{2}\left(1-\kappa \alpha^{-1}\right) X$ positive integers $N \leq X$ with $N \equiv \kappa(\bmod 2)$. This proves the forward implications of the statements in Theorem 1 . The reverse implications follow immediately from Theorems 2 and 3 combined with the lower bound of Proposition 2 and partial summation.

7. Remarks. For an irrational number $\alpha$ in the range $0<\alpha<1$, it is clear that the Beatty sequence $\mathcal{B}_{\alpha, \beta}$ contains all prime numbers. In this case, since $\psi^{(\kappa)}(x)=1$ for all $\kappa \geq 1$ and $x \in \mathbb{R}$, the statements in Theorems 2 and 3 are consistent with known results for the number of representations of an integer $N$ as a sum of $\kappa$ prime numbers.

It would be interesting to see whether the results of this paper can be extended to include irrational numbers $\alpha$ of infinite type (with a weakened error term).

To address a question that was posed in a preprint of the present paper, Kumchev [10] has studied representations of the form $N=p_{1}+\cdots+p_{k}$ with each prime $p_{j}$ lying in the Beatty sequence $\mathcal{B}_{\alpha_{j}, \beta_{j}}$, where the numbers $\alpha_{j}, \beta_{j}$ are real, each $\alpha_{j}$ is irrational (of finite type) and greater than one, and at least one ratio $\alpha_{i} / \alpha_{j}$ is irrational.

During the course of our investigations, we observed an interesting phenomenon. If $\alpha, \beta, \beta^{\prime} \in \mathbb{R}$ with $\alpha>1$ and $\alpha$ is an irrational number of finite type, put

$$
\mathcal{G}_{\kappa}(\alpha, \beta ; N)=\sum_{\substack{n_{1}+\cdots+n_{\kappa}=N \\ n_{1}, \ldots, n_{\kappa} \in \mathcal{B}_{\alpha, \beta}}} \Lambda\left(n_{1}\right) \cdots \Lambda\left(n_{\kappa}\right)
$$

as before, and let $\mathcal{G}_{\kappa}\left(\alpha, \beta^{\prime} ; N\right)$ be defined similarly. If $\beta^{\prime}=\beta+\alpha / \kappa$ for some fixed $\kappa>\alpha$, then it is easy to see that the Beatty sequences $\mathcal{B}_{\alpha, \beta}$ and $\mathcal{B}_{\alpha, \beta^{\prime}}$ contain different sets of primes. Nevertheless, by Theorem 3 one can immediately conclude that

$$
\mathcal{G}_{\kappa}(\alpha, \beta ; N) \sim \mathcal{G}_{\kappa}\left(\alpha, \beta^{\prime} ; N\right) \quad(N \rightarrow \infty) .
$$

\section{References}

[1] A. Balog and A. Perelli, Exponential sums over primes in an arithmetic progression, Proc. Amer. Math. Soc. 93 (1985), 578-582.

[2] W. Banks and I. Shparlinski, Prime numbers with Beatty sequences, preprint, 2006, http://arxiv.org/abs/0708.1015.

[3] Y. Bugeaud, Approximation by Algebraic Numbers, Cambridge Tracts in Math. 160, Cambridge Univ. Press, Cambridge, 2004.

[4] L. K. Hua, Introduction to Number Theory, Springer, Berlin, 1982. 
[5] M. N. Huxley, The Distribution of Prime Numbers. Large Sieves and Zero-Density Theorems, Clarendon Press, Oxford, 1972.

[6] H. Iwaniec and E. Kowalski, Analytic Number Theory, Amer. Math. Soc. Colloq. Publ. 53, Amer. Math. Soc., Providence, RI, 2004.

[7] A. Y. Khinchin, Zur metrischen Theorie der diophantischen Approximationen, Math. Z. 24 (1926), 706-714.

[8] N. M. Korobov, Exponential Sums and Their Applications, Math. Appl. (Soviet Ser.) 80, Kluwer, Dordrecht, 1992.

[9] L. Kuipers and H. Niederreiter, Uniform Distribution of Sequences, Pure Appl. Math., Wiley-Interscience, New York, 1974.

[10] A. Kumchev, On sums of primes from Beatty sequences, preprint, 2007, http://arxiv. org/abs/0706.0943.

[11] A. F. Lavrik, Analytic method of estimates of trigonometric sums by the primes of an arithmetic progression, Dokl. Akad. Nauk SSSR 248 (1979), 1059-1063 (in Russian); English transl.: Soviet Math. Dokl. 20 (1979), 1121-1224.

[12] K. K. Norton, Upper bounds for sums of powers of divisor functions, J. Number Theory 40 (1992), 60-85.

[13] K. F. Roth, Rational approximations to algebraic numbers, Mathematika 2 (1955), $1-20$.

[14] -, Corrigendum to "Rational approximations to algebraic numbers", ibid., 168.

[15] W. M. Schmidt, Diophantine Approximation, Lecture Notes in Math. 785, Springer, Berlin, 1980.

[16] I. M. Vinogradov, The Method of Trigonometrical Sums in the Theory of Numbers, Dover Publ., Mineola, NY, 2004.

[17] B. M. Wilson, Proofs of some formulae enunciated by Ramanujan, Proc. London Math. Soc. 21 (1922), 235-255.

[18] E. Wirsing, Das asymptotische Verhalten von Summen über multiplikative Funktionen, Math. Ann. 143 (1961), 75-102.

Department of Mathematics

University of Missouri

Columbia, MO 65211, U.S.A.

E-mail: bbanks@math.missouri.edu ahmet@math.missouri.edu nevans@math.missouri.edu

Received on 10.1.2007

and in revised form on 16.8.2007 